\title{
Fixed Retainers Vs Removable Retainers - Which Is Better?
}

\author{
Dr. N Tamilkkumaran BDS ${ }^{1}$, Dr. Sumathi Felicita MDS (Associate Professor) ${ }^{2}$ \\ ${ }^{I}$ (Department of Orthodontics, Saveetha Dental College, India) \\ 2 (Department of Orthodontics, Saveetha Dental College, India)
}

\begin{abstract}
Orthodontic retention is the maintenance of teeth in the ideal position after active orthodontic treatment. Success of treatment is measured by the orthodontist achieving the intended objectives, maintaining the teeth during retention and satisfying the patient. Retention can be achieved by various appliances. It can be divided into removable retention and fixed retention. The aim of the review is to find out if a fixed orthodontic retainer has better retention capability in post orthodontic treatment.
\end{abstract}

Keywords: Bonded retainer, Orthodontic retention, Relapse, Removable retainer, Stability

\section{Introduction}

Retention is a very important aspect in an orthodontic treatment. It's purely a waste of time if a perfectly done fixed orthodontic treatment is not stable after debonding. In orthodontics, retention can be defined as the phase of orthodontic treatment that attempts to keep teeth in their corrected position following orthodontic therapy, and is thus a very integral part of the treatment [1]. In cases, where proper retention was not gained during post treatment phase, relapse occurs. Relapse occurs mainly due to 3 major reasons, firstly thegingival and periodontal tissues are affected by orthodontic tooth movement and require time for reorganization when the appliances are removed, second-the teeth may be in an inherently unstable position after the treatment, so that soft tissue pressures constantly produce a relapse tendency, and last but not the least the changes produced by growth may alter the orthodontic treatment result [2]. The next most frequently asked question would be how long is the recognized duration of retention? There is no ideal duration of retention period, however in relation to periodontal factors. It takes a minimum of 232 days for fibers around the teeth to remodel to the new position [3]. To minimize the incidence of relapse it is vital for the all patients who have orthodontic treatment to use some type of retainer [1].

Retention can be achieved by various appliances which can be broadly classified as removable appliance and fixed appliance. Hawley's appliance and Essix retainers are types of removable appliance which have been widely used. However, Hawley's appliance is the most commonly used removal appliance [4]. Hawley's appliance consists of a palatal portion made of acrylic and a labial bow made of 0.020 to 0.036 inch stainless steel wire, whereas Essix retainer typically consist of a 0.030 inch plastic, which completely covers all surfaces of the teeth. Fixed retainers are normally used in situations whenever intra-arch instability is anticipated and prolonged retention is intended for a patient [2][5]. It was first proposed by Zachrisson [6] in 1977, where he used individually adjusted, multi-stranded wire bonded on to the lingual surface of each tooth for long term retention. There are various forms of fixed retainers. The most frequently used are the mandibular canine to canine (3-3) bonded retainer bar ( 0.030 or $0.032 \mathrm{inch}$ ) and the thin $0.0215 \mathrm{inch}$, flexible retainers and spiral wire retainers [1] [7] [8]. Adjunctive procedures such as interproximal stripping [9] and circumferential supracrestal fiberotomy [10] [11] can also be applied to the teeth or surrounding periodontium to aid the retention process. This review of literature will mainly focus on how fixed retainers play a vital role in post orthodontic retention.

\section{History}

In 1965, Newman was the first to report the use of direct bonding of orthodontic attachments to the tooth surface [12], whereas Kneirim was the first to report the use of bonded fixed retainers [13]. In 1977, Zachrisson published the possible benefits of using multistranded wires instead of the earlier use of round orthodontic wire for constructing the bonded retainers [6]. Later in 1982, Årtun and Zachrisson came up with a clinical technique. This technique involves bonding the wire only on the canine teeth [14]. Zachrisson proposed the advantages of bonding the multistranded wire on all the teeth in the labial aspect [14]. The proponents of multistranded wire claim its has two major advantages: a) increased mechanical retention for composite with no need of retentive loops, b) allowance of physiologic movement of teeth in spite of bonding several adjacent teeth due to its flexibility. As an alternative to multistranded wire, the uses of resin fiberglass strips have been developed. 


\section{Fixed retainer vs Removable retainer}

Segner et al (2000) performed a retrospective study which assessed the reliability of bonded retainers. Among his results, he reports that these retainers were not responsible for causing caries and that the majority of retainer breakages or losses occurred in the first 3 to 6 months. More importantly he concluded that bonded retainers were highly competent and dependable in maintaining tooth alignment. This study has the largest sample size of contributing to a fair level of evidence. However because of the lack of a control group it is not possible to established whether due to the bonded retainers preventing tooth movement or because of absence of attempted movement by the teeth themselves [15].

Artun et al (1997) carried out a study comparing 4 types of retainers. Subjects were divided into 4 groups, 3 groups receiving each a different version of bonded retainer while the $4^{\text {th }}$ group received a removable retainer and served as the control group. His paper reported that the 4 types of retainers were equally capable of maintaining incisor positioning. This study is considered to be a randomized-controlled trial, and with such a strong design it has the highest level of evidence. However there is at the same time the lack of a true control group that would have ideally received no means of retention at all [16].

Edwards (1988) investigated a less prescribed means of retention which involves a surgical process where a B.P. blade is inserted into the gingival sulcus severing the epithelial attachment surrounding the teeth as well as the transeptal fibers. The study divided subjects into 2 groups, one in which circumferential supracrestal fiberotomy procedure and removable retainer worn at night was given, and another were only a removable retainer was worn which to served as a control. Although the randomization process was done inappropriately (alternating assignment of subjects to the 2 groups) thereby granting the study only a fair level of evidence, the study reports that the circumferential supracrestal fiberotomy procedure was more efficient than a removable retainer alone especially in preventing purely rotational relapse [17].

Little et al reported that long term retention is recommended and the better choice is bonded retainer for prevention of recurrence of crowding in the mandibular anterior segment [18] [19].

Watted et al investigated the effect of mandibular canine to canine lingual retainers bonded to 2 to 6 teeth for incisor mobility. With a total of 60 participants, divided into 3 equal groups ( 2 groups with mandibular bonded retainers and one control group with removable retainers), the study results revealed that tooth mobility decreased with the number teeth bonded to the retainer [20].

Stormann et al in prospective randomized study compared 2 types of fixed mandibular retainers. In total 103 patients had either canine to canine (bonded to 6 teeth) or canine and canine (bonded to 2 teeth). Using little's irregularity index to measure relapse over a period of 24 months, it was found that canine to canine bonded retainer had a greater degree of stability whereas the canine and canine removable retainer had were associated with frequent relapse as the incisors were not bonded [21].

\section{Conclusion}

As a conclusion orthodontic retention is one of the most important criteria in achieving successful treatment results. It is proven that fixed retainers are a better choice to retained newly aligned teeth in the corrected position compared to removable retainers.

\section{References}

[1] Littlewood SJ, Millett DT, Doubleday B, Bearn D, Worthington HV. Retention procedures for stabilizing tooth position after treatment with orthodontic braces. Cochrane Database Syst. Rev. 2004; (1): CD00SS83 Review.

Proffit WR, and Fields HW. Contemporary Orthodontics, (New York: Mosby Inc, 2005).

[3] Reitan K. Clinical and histologic observations on tooth movement during and after orthodontic treatment. Am J Orthod 1967; 53: $721-45$.

[4] Hawley, C. A. (1919). "A removable retainer." Int J Ortod. Oral Surg.(5): 291-305.

[5] Zachrisson, BU: Long-term experience with bonded retainers: update and clinical advice. J Clin Orthod. 41, 2007, $728-737$.

[6] Zachrrison B U 1977 Clinical experience with direct-bonded orthodontic retainers . American Journal of Orthodontics 71: 440-448

[7] Zachrisson B U, Büyükyilmaz T Bonding in orthodontics . In: Graber L W (ed). Orthodontics: current principles and techniques, ( $4^{\text {th }}$ ed. Mosby , St Louis , 2005) pp. $621-659$

[8] Littlewood S J, Millett D T, Doubleday B, Bearn D R, Worthington H V 2006 Retention procedures for stabilising tooth position after treatment with orthodontic braces. Cochrane Database of Systematic Reviews 1:CD002283

[9] Boese LR. Fiberotomy and reproximation without lower retention, nine years in retrospect: Part I. Angle Orthod 1980; 50: 88-97.

[10] Edwards, JG. A surgical procedure to eliminate rotational relapse. Am J Orthod 1970; 57: 35-46.

[11] Edwards JG. A long-term prospective evaluation of the circumferential supracrestal fiberotomy in alleviating orthodontic relapse. Am J Orthod Dentofacial Orthop 1988; 93:380-7.

[12] Newman GV. Epoxy adhesives for orthodontic attachments: progress report. American Journal of Orthodontics. 1965;51(12):90112.

[13] Knierim R. Invisible lower cuspid to cuspid retainer. The Angle Orthodontist. 1973;43(2): 218-20

[14] Zachrisson B. The bonded lingual retainer and multiple spacing of anterior teeth. Swed Dent J Suppl. 1982;15:247-55.

[15] Segner D, Heinrici B. Bonded Retainers: Clinical Reliability. Journal of Orofacial Orthopedics 2000; 61:325-328

[16] Artun J, Spadafora AT, Shapiro PA. A 3-year follow-up study of various types of orthodontic canine-to-canine retainers. European Journal of Orthodontics 1997; 19(5): 501-9. 
[17] John GE. A long-term prospective evaluation of the circumferential supracrestal fiberotomy in alleviating orthodontic relapse. American Journal of Dentofacial Orthopedics 1988; Vol 93(5):380-387.

[18] Little R M, Riedel R A, Årtun J 1988 An evaluation of changes in mandibular anterior alignment from 10 to 20 years postretention American Journal of Orthodontics and Dentofacial Orthopedics $93: 423-428$

[19] Little R M , Wallen T R, Riedel R A 1981 Stability and relapse of mandibular anterior alignment - first premolar extraction cases treated by traditional edgewise orthodontics American Journal of Orthodontics $80: 349-365$

[20] Watted, N.M. Wieber et al 2001 Comparison of incisior mobility after insertion of canine-to-canine lingual retainers bonded to two or to six teeth. A clinical study. J Orofac Orthop 62(5): 387-396

[21] Stormann, I and E. Ulrike 2002 "A prospective randomized study of different retainer types" Journal Of Orofacial Orthopaedics 63: $42-50$ 